\title{
Research on the construction of information resources sharing mechanism in the network environment
}

\author{
Yihua Deng and Senglin Lu \\ Jiangxi Science \& Technology Normal University, Nanchang,Jiangxi ,China
}

330013

Key words: network environment; sports; information resources; sharing mechanism; thinking

Abstract: with the development of network technology to further deepen, for the construction of sports information resources sharing system has laid a technical foundation. But in order to successfully built sports information resources efficient, reliable, advanced and rich sharing system, comprehensive planning, strict organization, raise funds and develop collection channels, create good software and hardware environment and the introduction of competition and incentive mechanism so as to realize the sharing of sports information resources, the maximum value of good the social and economic benefits.

Exploration of sports information resources in the success of "competition and incentive" sharing mechanism construction is based on the network environment of the modern information resources transmission platform, the implementation of various types of sports information resources integration application sharing information resources system, for better development

Laying a solid foundation. Carrying out the sports information resources and applied research, relevant personnel can use the sports information resources sharing mechanism to obtain the required information for all kinds of sports resources, sports management work, scientific research and practical work of sports service.

1 the foundation of the construction of "competition and incentive" sharing mechanism of sports information resources

1.1 the maturity and perfection of the processing technology of sports information resources

With the development of modern network technology, multimedia technology and big data technology, it provides a strong technical support for the sharing of sports information resources. At present, the Internet has penetrated into every aspect of social life, has become an indispensable part of people's daily life, especially for people in the hands of essential products, mobile phone has a powerful information processing capabilities and improve the network information terminal receiving function, sports information resources users through the network platform to facilitate access to resources it is possible, with hardware and software environment conditions are ripe, and the user terminal system with the improvement and innovation of related technology is still in the continuous optimization process, will help the sports information resources users can efficiently obtain various categories of sports information resources.

1.2 universal application of digital technology. The use of information network environment, mature technology, digital transmission and storage technology, will enable the sports information resource digitization has become a reality, and also for the realization of sports information resources sharing foundation. To support big data technology, multimedia technology and network technology, the construction of science or the introduction of sports information resources "competition and incentive" sharing mechanism has become a top priority for the sports management department, must do a good job. At present, our country and the world, the research of sports information resources 
"competition and incentive" sharing mechanism has made considerable achievements, these achievements have been transformed into a guiding theory guide sports information resources sharing. Based on the above environment, how to further improve the level of sports information resources service society, has become the core task of information resources sharing and construction workers. In China, for example, in the State Administration under the leadership is to create the sports information resources at all levels of sharing platform, has laid a solid material foundation for the sports information resources system construction of high quality.

1.3 sports information resources sharing mechanism research content. It covers all aspects of the field of sports, sports information resources sharing mechanism construction of science, its goal is to require the integration of high quality sports information resources more, each field of sports involved into the sports information resources construction within the scope of. Only in this way, the establishment of sports information resources sharing mechanism, in order to achieve the sharing of sports information resources to maximize the. Therefore, the top-level design in the planning of sports information resources sharing mechanism must take into account the content of sports information resources sharing must be fully covered. Only in this way, the construction of the sports information resources sharing mechanism has fresh vitality and practical significance, in order to meet the needs of the community to share all levels of sports information resources.

1.4 sports information resources are very rich. The source of sports information resources are available in a variety of sports resources in the international level, can also be a variety of sports resources in the domestic level, information can be various forms of sports world level, can also be the domestic ordinary sports competition information, thus the source of rich variety. Sports information resources in general, with a wide distribution, the content of the basic features, such as distributed existence. As sports information resources involved in sports, the kind of aspect, the content is very rich.

2 sports information resources sharing is still a problem

\section{1 lack of planning}

Although our country is the State Sports General Administration of unified leadership of China's sports and sports information resources macro management. However, in our country sports information resources sharing construction has not formulated the unified planning, also does not have the construction into the unified sports information resources sharing big database. It is understood and access to a large number of relevant information that the construction of sports information resources in our country is still in a spontaneous, fragmented and non unified stage, restricted the development of sports information resources sharing.

\section{2 insufficient funds}

To carry out the construction of sports information resources sharing, the need to have sufficient funds to invest. However, in real life, people affected by the lack of knowledge, to carry out the top-level design, sports information resources sharing is not unified planning, the national level there is no natural capital investment, directly affect the development of sports information resources sharing. It is well known that the modern information resources construction project, is the need for a large amount of capital investment in order to work properly. There is no stable capital investment, the construction of sports information resources sharing will be affected. At present, the information resources sharing of sports information resources in our country is at a low level.

2.3 standard is not complete

As the development speed of information is more than people's imagination, a lot of 
standardized information resources sharing construction standards to research and development, can not meet the needs of the construction of sports information resources sharing. Hence the lack of coordination phenomenon will be not at all surprising, the higher authorities need as soon as possible to develop standardized criteria to unify the current chaotic situation.

\section{4 team is not strong}

To carry out the construction of sports information resources, it is necessary to build a team of professional and technical personnel with high quality. To this end, the construction units, the need to strengthen the introduction and training of personnel, special attention should be paid to meet the computer skills and network information technology personnel training. It is understood the lack of these compound talents. In short, to successfully complete the construction of sports information resources, the first to solve the problem of the construction of the above personnel and reserves.

3 sports information resources sharing mechanism construction

3.1 institutions to build

To organize the construction of a powerful information resources sharing system, the most important point is the construction of institutions. Only the establishment of a sound organizational structure in order to provide protection for the construction of sports information resources system. Only by establishing the organization to effectively organize the sports information resources sharing mechanism construction work, and macro-control of the entire sports information resources system, comprehensive integration of sports information resources in various forms, strengthening its resource system, finally realizes the sports information resources sharing target.

\section{2 mechanism construction}

Sports information resources sharing needs multi support, multi talent cooperation and close cooperation in all levels of system engineering. First of all, need to do preliminary research work, fully demonstrated the sports information resources sharing mechanism construction scheme is feasible, scientific and practical; secondly, to develop the implementation plan, and advance the practice plan is feasible, the result is reliable and is easy to modify and perfect; third, the introduction of mechanisms can play a role, whether the main assessment the mechanism of lead elements of competition benefits only can exist only elements of competition and stimulate innovation potential of staff. At the same time, the incentive elements are incorporated into the service to improve their work efficiency. The introduction of competition and incentive elements in order to develop a scientific mechanism for the sharing of sports information resources.

\section{3 construction standards}

Unified sports information resources construction standards, the purpose is to better achieve the goal of sports information resources sharing. Only in strict accordance with the requirements of standardization, to carry out the construction of sports information resources, in order to achieve the desired goal. The formulation of the standardization construction standards must be completed by the national level, because of its influence, significance and value. Must pay attention to the organization of professional and technical personnel and other forces, making the correct metadata, data, software and hardware security domain valve and the process of technical standards, lay the technical foundation and integration of various categories of sports information resources for digital sports information resource system has become the digital information resources.

\section{4 resource integration}

Effective integration of sports information resources is the foundation of realizing the sharing of information resources of sports information. One should increase the collection of sports information 
resources, to multi-channel, multi-channel and multi-level sports information resources to collect complete. For various types of information resources and will be arranged to collect the effective integration of illustrations, characteristics, the results have strong readability and practicability. According to the characteristics and needs of sports information resources, and actively build a special sports resources information resources database. In order to achieve the ultimate content refinement, high quality sports information resources system, a large number of non use value, no preservation of the value of sports information resources must be cleared. Actively carry out the work of purification of useless information resources, as far as possible to clear the redundant information resources.

\section{5 funding}

To become bigger and stronger, and to do a good job of sharing information resources system with the characteristics of sports, we must have sufficient funds to ensure. Raising enough funds is also a very important task in the construction of the information resources sharing system of sports activities. To actively carry out promotional activities, for the community to support technology, to explore the source of funds and to develop effective capital inflow mechanism, the use of all available power, to expand the source of the channel.

3.6 incentive mechanism

The successful construction of sports information resource sharing system, we must introduce competition and incentive mechanism, at the same time, the corresponding supporting should also formulate relevant measures and examination system and evaluation system, in order to truly build and perfect the scientific competition mechanism and incentive mechanism, to ensure the sports information resources sharing system construction work smoothly.

In a word, to establish and perfect the system of sports information resources sharing, we must lay a solid resource base, formulate measures feasible, establish the corresponding work system, the relevant departments and actively seek the support of the leadership and the construction of sufficient funds. To make reasonable and feasible "competition and incentive" mechanism, to ensure the technology, human resources and resources, to build a sports information resources sharing mechanism, to maximize the sharing of sports information resources.

\section{Reference}

[1] Lu forest. Thinking about the construction of University Archives Website Based on network environment [J]. Shanxi archives 2014 (6);

[2] Deng Ping. Study on the special topic database of Guangzhou sports information resources construction and practice of the [J]. library, 2011 (10);

[3] Lei Jun Zeyong, travel. [J]. technical information development and economy research on the mechanism of the integration and sharing of university sports information resources, 2013 (9);

[4] in the digital era of sports information resources to optimize the integration of [J]. electronic testing, 2015 (14). 